\title{
Impact of MRI Selection on Triage of Endovascular Therapy in Acute Ischemic Stroke: The MRI in Acute Management of Ischemic Stroke (MIAMIS) Registry
}

\author{
Kunakorn Atchaneeyasakul ${ }^{a}$ Ty Shang ${ }^{a}$ Diogo Haussen ${ }^{a}$ Gustavo Ortiz ${ }^{a}$ \\ Dileep Yavagal ${ }^{\mathrm{a}, \mathrm{b}}$ \\ aDepartment of Neurology, University of Miami Miller School of Medicine, Miami, FL, USA; \\ ${ }^{b}$ Department of Neurological Surgery, University of Miami Miller School of Medicine, \\ Miami, FL, USA
}

\section{Keywords}

Acute ischemic stroke $\cdot$ Endovascular therapy $\cdot$ Mismatch $\cdot$ MRI

\begin{abstract}
Background: The recently published multicenter randomized DAWN trial confirmed greater outcome benefit of endovascular therapy (ET) for anterior circulation large vessel occlusion ischemic stroke from 6 to $24 \mathrm{~h}$ from symptom onset compared to medical management in patients selected by advanced imaging with MRI or perfusion CT to identify mismatch between clinical deficit and infarct volume, which represents salvageable penumbra. The debate of CT over MRI is usually the potentially increase time consumption and the difficulty in establishing an adequate standardized workflow utilizing MRI during the hyperacute phase. Purpose: While CT-based selection of patients is the current standard of care, we sought to determine the time impact of the alternative approach of MRI selection in the $0-12 \mathrm{~h}$ window. Methods: In the MRI in Acute Management of Ischemic Stroke (MIAMIS) registry, we retrospectively analyzed 89 consecutive patients from January 2008 to January 2010 who presented with acute stroke symptoms with a National Institutes of Health Stroke Scale score $\geq 5$ or aphasia within $0-12 \mathrm{~h}$ from symptom onset. The presence of penumbra was determined by MR perfusion-diffusion mismatch or clinical diffusion mismatch. Patients were stratified based on the presence of mismatch and clinical outcomes in patients who received ET. Imaging times
\end{abstract}

K. Atchaneeyasakul and T. Shang contributed equally to the manuscript.

Dileep Yavagal, MD, MBBS

University of Miami Miller School of Medicine

1120 NW 14th Street

Miami, FL 33136 (USA)

E-Mail dyavagal@ med.miami.edu 
were recorded. Results: The MRI turnaround time was $95.5 \pm 48.5 \mathrm{~min}$. The total MRI time was $27.7 \pm 12.8 \mathrm{~min}$. Seventeen (19.1\%) patients were found to have nonvascular etiology. Mismatch was found in 35 (48.6\%) patients with acute ischemic stroke (AIS). Patients with nonvascular etiology were younger (55.7 vs. 65.6 years, $p<0.02$ ), without any vessel occlusion or mismatch noticed in this group. We dichotomized the 39 AIS patients with vessel occlusion into two subgroups: these with mismatch and these without. Patients without mismatch were older (76.7 vs. 64.4 years, $p<0.05)$, more likely to have congestive heart failure $(71.4 \mathrm{vs.} 22 \%$, $p<0.03$ ), a higher total serum cholesterol level (196 vs. $156 \mathrm{mg} / \mathrm{dL}, p<0.04$ ), and medium to large lesions on diffusion-weighted imaging (DWI) (85.7 vs. $37.5 \%, p<0.04)$. Conclusions: Multimodality MRI screening for AIS symptoms for ET is feasible. Optimizing each center's protocol and the utilization of MRI with DWI only may be a time-saving alternative.

(c) 2019 S. Karger AG, Basel

\section{Introduction}

Stroke is a leading cause of morbidity and mortality worldwide. Multiple randomized studies have shown that mechanical thrombectomy in anterior circulation large vessel occlusion ischemic stroke has led to a significant improvement in functional outcome compared to intravenous tissue plasminogen activator (tPA) alone when it is performed within $6 \mathrm{~h}$ from symptom onset [1]. Since its first introduction about 30 years ago, penumbra in acute ischemic stroke (AIS) has been the target of diagnosis and treatment [2]. Penumbra was classically defined as hypoperfused tissue surrounding the ischemic core in which blood flow is too low to maintain electric activity but sufficient to preserve ion. MRI has been proposed as the method for evaluating penumbra and can be useful for endovascular therapy (ET) patient selection. There have been multiple retrospective trials utilizing advanced imaging with MRI in patient selection for ET beyond the $6 \mathrm{~h}$ from symptom onset [3]. The recently published multicenter randomized DAWN trial (diffusion-weighted imaging [DWI] or perfusion CT assessment with Clinical Mismatch in the Triage of Wake Up and Late Presenting Strokes Undergoing Neurointervention with Trevo) have confirmed greater outcome benefit of ET from 6-24 h of symptom onset compared to medical management in patients selected by advanced imaging with MRI or perfusion CT to identify mismatch between the clinical deficit and infarct volume [4]. The clinical and imaging mismatch seen is likely due to the variable presence of salvageable penumbra, which could be visualized by advanced neuroimaging, based on collateral supply in individual patients rather than solely on time from symptom onset.

The DAWN trial laid the groundwork for an acute imaging protocol in patient selection for stroke ET. In the trial MRI, DWI, or perfusion CT were used to identify clinical and imaging mismatch. CT and MRI can provide similar information, but both show respective advantages and disadvantages. The debate over MRI is usually the potentially increased time consumption and the difficulty in establishing an adequate standardized workflow utilizing MRI during the hyperacute phase [5]. Kang et al. [6] demonstrated that the door to needle time for MRI screening is significantly longer than that for CT screening for AIS patients treated with intravenous tPA.

While CT-based selection of patients is the current standard of care, we sought to determine the impact of the alternative approach of MRI selection in the $0-12 \mathrm{~h}$ window. We determined the impact on imaging start to puncture, proportion of patients excluded from ET, and stroke mimics. 


\begin{tabular}{l|l}
\hline DOI: 10.1159/000490580 & $\begin{array}{l}\text { C 2019 S. Karger AG, Basel } \\
\text { www.karger.com/ine }\end{array}$ \\
\hline
\end{tabular}

Atchaneeyasakul et al.: MRI Selection for Thrombectomy: The MIAMIS Registry

\section{Methods}

\section{Patient Selection}

Since January 2008, our center has instituted a clinical policy of emergent MRI with diffusion, perfusion, FLAIR, gradient echo, and MR angiogram of the circle of Willis in patients with AIS with a National Institutes of Health Stroke Scale (NIHSS) score $\geq 5$ or aphasia within $12 \mathrm{~h}$ from symptom onset according to the IRBapproved MRI in Acute Management of Ischemic Stroke (MIAMIS) Registry. Patients with perfusion-diffusion mismatch (PDM) or clinical diffusion mismatch (CDM), as assessed by a stroke neurologist, were triaged to ET. Patients received intravenous tPA prior to ET if indicated. We retrospectively analyzed the medical records of all consecutive AIS patients who met the clinical protocol criteria for emergent MRI from January 2008 to January 2010. PDM was defined by the presence of a perfusion-weighted imaging (PWI) volume measuring $>20 \%$ of the DWI volume based on the DEFUSE study [7]. CDM was defined subjectively as the presence of clinical deficit disproportionately more severe than the DWI lesions by the estimation of a stroke neurologist. Time to treatment was defined as the time from stroke onset to groin puncture time. MRI turnaround time was defined as the time from MRI order to completion.

\section{Imaging Methods}

A CT scan of head without contrast was acquired with contiguous 5-mm-thick axial sections (140 kV, $299 \mathrm{mAs}$ ). CT was performed in all patients prior to MRI. MRI scans were performed on two 1.5-Tesla imaging systems (Avanto, Siemens, Erlangen, Germany and Infineon, Phillips, Eindhoven, The Netherlands). The MRI sequences included FLAIR (TE/TR $140 \mathrm{~ms} / 10 \mathrm{~s}$, slice thickness $5 \mathrm{~mm}$, interslice gap $1 \mathrm{~mm}$, FOV $220 \times$ $220 \mathrm{~mm}^{2}$ ), DWI (TE/TR $80-110 \mathrm{~ms} / 5 \mathrm{~s}$, slice thickness $5 \mathrm{~mm}$, interslice gap $1 \mathrm{~mm}$, FOV $220 \times 220 \mathrm{~mm}^{2}$, b value $1,000 \mathrm{~s} / \mathrm{mm}^{2}$ ), and PWI (TE/TR $40 \mathrm{~ms} / 1,500 \mathrm{~ms}$, slice thickness $5 \mathrm{~mm}$, interslice gap $1 \mathrm{~mm}$ ). Cerebral blood volume, cerebral blood flow, and mean transit time were calculated with the arterial input function derived from the middle cerebral artery (MCA) ipsilateral to the infarct. An apparent diffusion coefficient map was generated on the scanner console at the time of imaging. The MRI technologist was available in-house at all time.

\section{AIS Therapies}

Intravenous tPA was given at $0.9 \mathrm{mg} / \mathrm{kg}$ as full dose or $0.6 \mathrm{mg} / \mathrm{kg}$ as bridging dose $(10 \%$ bolus, $90 \%$ continuous infusion over $1 \mathrm{~h}$, up to a maximum of $90 \mathrm{mg}$ ) to patients who met the criteria. Cerebral angiogram and appropriate treatment were performed in patients who were triaged to ET. As this patient cohort was treated prior to 2010, mechanical thrombectomy was not done with the conventional stent retriever method, and multiple devices including the MERCI Retrieval System and the Penumbra Aspiration System were applied.

\section{Clinical and Imaging Scoring}

The NIHSS score was used to evaluate the neurological status of patients with acute stroke symptoms on presentation. The DWI lesions were visually classified into none to small if no lesion was seen on DWI or if the lesion was smaller than half the size of the thalamus, and medium to moderate if the lesion was larger but less than one-third of the MCA territory.

Outcome Analysis

Good outcome was defined by a 90-day modified Rankin Scale score $\leq 2$. Successful recanalization was defined by modified TICI $2 \mathrm{~b}$ or 3 . The rate of symptomatic intracranial hemorrhage was assessed as a safety endpoint on follow-up CT.

\section{Statistical Analysis}

Statistical data analysis was performed with the SPSS software (SPSS Inc., Chicago, IL, USA). Categorical variables were analyzed using the two-tailed $\chi^{2}$ test or the Fisher exact test. Continuous variables were analyzed using the independent $t$ test. The Mann-Whitney $\mathrm{U}$ test was used for median analysis. Multiple logistic regression was performed for hypothesis testing. Statistical significance was considered at $p<0.05$. 


\section{Results}

\section{MRI Workflow Time}

A total of 89 patients met our study criteria; $68.5 \%$ of the cases were enrolled by PDM criteria and the rest by CDM criteria. The average time from symptom onset to MRI was $308 \pm$ $144 \mathrm{~min}$. The MRI turnaround time was $95.5 \pm 48.5 \mathrm{~min}$. The total MRI time was $27.7 \pm 12.8 \mathrm{~min}$. The order to first MRI time was $73.8 \pm 51.95 \mathrm{~min}$.

\section{Stratification of Patients Based on Vascular and Nonvascular Etiology}

A total of 89 patients met our study criteria. Seventeen (19.1\%) patients were found to have nonvascular etiology (Table 1$)$. The most common diagnoses were seizures $(5 / 17,29.4 \%)$ and conversion disorder $(4 / 17,23.5 \%)$. The remaining diagnoses included unmasking of prior stroke deficits $(2 / 17,11.8 \%)$, sepsis $(1 / 17,5.9 \%)$, migraine $(1 / 17,5.9 \%)$, progressive multifocal leukoencephalopathy $(1 / 17,5.9 \%)$, and unknown $(2 / 17,11.8 \%)$. Figure 1 shows the allocation of patients with AIS symptoms within the first $12 \mathrm{~h}$ from symptom onset included in the study triaged for ET.

Compared with patients with vascular etiology (AIS), patients with nonvascular etiology were younger ( 55.7 vs. 65.6 years, $p<0.02$ ). No vessel occlusion or mismatch was noticed in the nonvascular etiology group. There was no statistical difference in the remainder of the variables compared.

In patients with vascular etiology, the median admission NIHSS score was 15.5 (IQR 9-20). Vessel occlusion and mismatch were noticed in 39 (54.2\%) and 35 (48.6\%) patients, respectively. Intravenous tPA was administered in 28 (38.9\%) patients.

\section{Stratification of Patients with AIS Based on the Presence of Mismatch}

Among the patients with AIS, 35 (48.6\%) were found to have mismatch (Table 2). Patients with mismatch had higher admission NIHSS scores (17 vs. 11, $p<0.02$ ). Vessel occlusion (91.4 vs. $18.9 \%, p<0.001$ ) and atrial fibrillation (42.8 vs. $18.9 \%, p<0.03$ ) were more frequently noticed in patients with mismatch. Serum levels of triglyceride (100.9 vs. $155.2 \mathrm{mg} / \mathrm{dL}, p<0.01$ ) and cholesterol (161 vs. $186 \mathrm{mg} / \mathrm{dL}, p<0.04$ ) were significantly lower in patients with mismatch.

There were fewer patients with mismatch who had an NIHSS score $\leq 5$ ( 2.87 vs. $21.6 \%, p<$ $0.03)$. Accordingly, there were more patients with mismatch who received intravenous tPA (51.4 vs. $27 \%, p<0.04)$. There were more patients with mismatch evaluated by PDM than patients without mismatch ( 85.7 vs. $56.8 \%, p<0.01)$. There were no statistical differences in the remainder of the variables compared.

We dichotomized the 39 AIS patients with vessel occlusion into two subgroups: those with mismatch and those without (Table 3). Patients without mismatch were older (76.7 vs. 64.4 years, $p<0.05$ ), more likely to have congestive heart failure (CHF) ( 71.4 vs. $22 \%, p<0.03$ ), and had a high total serum cholesterol level (196 vs. $156 \mathrm{mg} / \mathrm{dL}, p<0.04)$ and medium to large DWI lesions (85.7 vs. $37.5 \%, p<0.04$ ). There were no statistical differences in the remainder of the variables compared.

\section{Outcome of Mechanical Thrombectomy}

Among the patients with mismatch, 25 (71.4\%) were triaged to receive ET. This patient cohort was treated prior to 2010, so ET was not done with the conventional stent retriever method, and multiple devices including the MERCI Retrieval System and the Penumbra Aspiration System were applied. Seven (28\%) patients had good outcome. These patients were significant younger ( 49.4 vs. 66.7 years, $p<0.02$ ) and had less histories of hypertension $(29 \mathrm{vs.} \mathrm{89 \% ,}$ $p<0.01$ ) and a shorter time interval from MRI to recanalization (148 vs. $221 \mathrm{~min}, p<0.05$ ). The successful recanalization rate was $64 \%$. The symptomatic intracranial hemorrhage rate was $8 \%$. 


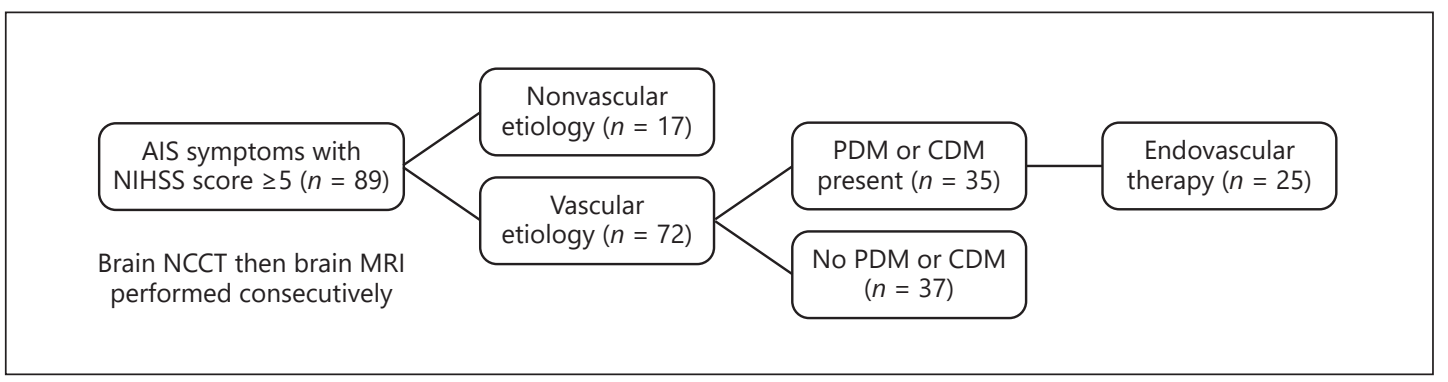

Fig. 1. Patients with AIS symptoms within the first $12 \mathrm{~h}$ from symptom onset included in the study triaged for endovascular therapy. AIS, acute ischemic stroke; CDM, clinical diffusion mismatch; NCCT, noncontrast CT; NIHSS, National Institutes of Health Stroke Scale; PDM, perfusion-diffusion mismatch.

Table 1. Comparison of baseline clinical and demographic variables among patients with vascular and nonvascular etiology

\begin{tabular}{lccc}
\hline & Vascular & Nonvascular & Significance \\
\hline Number of patients & 72 & 17 & \\
Age, years & $65.6 \pm 14.8$ & $55.7 \pm 17$ & $0.018^{\mathrm{a}}$ \\
Male sex & $52.8 \%$ & $41.1 \%$ & $0.39^{\mathrm{b}}$ \\
Median admission NIHSS score & $15.5(9-20)$ & $11(7.5-15.5)$ & $0.171^{\mathrm{c}}$ \\
Hypertension & $80.5 \%$ & $76.5 \%$ & $0.741^{\mathrm{d}}$ \\
$\quad$ Admission systolic BP, mm Hg & $155 \pm 30$ & $159 \pm 38$ & $0.654^{\mathrm{a}}$ \\
$\quad$ Admission diastolic BP, mm Hg & $89 \pm 18$ & $98 \pm 25$ & $0.09^{\mathrm{a}}$ \\
Diabetes mellitus & $37.5 \%$ & $35.2 \%$ & $0.866^{\mathrm{b}}$ \\
$\quad 137 \pm 62$ & $129.6 \pm 60$ & $0.658^{\mathrm{a}}$ \\
Admission glucose level, mg/dL & $62.5 \%$ & $47 \%$ & $0.243^{\mathrm{b}}$ \\
$\quad 104 \pm 41$ & $90 \pm 35$ & $0.251^{\mathrm{a}}$ \\
$\quad$ LDL, mg/dL & $44.7 \pm 18$ & $43.3 \pm 14$ & $0.799^{\mathrm{a}}$ \\
$\quad 128.8 \pm 76$ & $94 \pm 31$ & $0.109^{\mathrm{a}}$ \\
$\quad$ Triglyceride, mg/dL & $173.9 \pm 47.8$ & $152 \pm 38$ & $0.127^{\mathrm{a}}$ \\
Coronolesterol, mg/dL & $15.3 \%$ & $23.5 \%$ & $0.474^{\mathrm{d}}$ \\
Congestive heart failure & $19.4 \%$ & $5.9 \%$ & $0.285^{\mathrm{d}}$ \\
Atrial fibrillation & $30.5 \%$ & $5.9 \%$ & $0.061^{\mathrm{d}}$ \\
Intravenous tPA rate & $38.9 \%$ & $0 \%$ & $0.002^{\mathrm{b}}$ \\
Time from stroke onset to MRI, min & $318 \pm 151$ & $267.6 \pm 100$ & $0.197^{\mathrm{a}}$ \\
MRI turnaround time, min & $92 \pm 45$ & $108.6 \pm 60$ & $0.218^{\mathrm{a}}$ \\
Mortality & $9.7 \%$ & $0 \%$ & $0.338^{\mathrm{d}}$ \\
Mismatch & $35(48.6 \%)$ & 0 & $0.000^{\mathrm{b}}$ \\
Vessel occlusion & $39(54.2 \%)^{\mathrm{f}}$ & 0 & $0.000^{\mathrm{b}}$ \\
Perfusion-diffusion mismatch & $70.8 \%$ & $58.8 \%$ & $0.337^{\mathrm{b}}$ \\
Clinical diffusion mismatch & $29.2 \%$ & $41.2 \%$ & $0.002^{\mathrm{d}}$ \\
Endovascular therapy & $34.7 \%$ & $0 \%$ & \\
\hline
\end{tabular}

BP, blood pressure; HDL, high-density lipoprotein; LDL, low-density lipoprotein; NIHSS, National Institutes of Health Stroke Scale; tPA, tissue plasminogen activator. ${ }^{a}$ Student $t$ test. ${ }^{b} \chi^{2}$ test. ${ }^{c}$ Mann-Whitney U test. ${ }^{d}$ Fisher exact test. ${ }^{\text {e }}$ Ejection fraction $\leq 45 \%$. ${ }^{\mathrm{f}}$ One patient had severe stenosis of $>99 \%$ measured by cerebral angiography. 
Table 2. Comparison of baseline clinical and demographic variables among AIS patients with and without mismatch

\begin{tabular}{lccc}
\hline & Mismatch & No mismatch & Significance \\
\hline Number of patients & 35 & 37 & \\
Age, years & $64.6 \pm 15$ & $66.5 \pm 14$ & $0.597^{\mathrm{a}}$ \\
Male sex & $57 \%$ & $48.6 \%$ & $0.471^{\mathrm{b}}$ \\
Median admission NIHSS score & $17(11-20)$ & $11(6-18)$ & $0.011^{\mathrm{c}}$ \\
Hypertension & $77 \%$ & $83.4 \%$ & $0.477^{\mathrm{b}}$ \\
$\quad$ Admission systolic BP, mm Hg & $159 \pm 30$ & $152 \pm 30$ & $0.345^{\mathrm{a}}$ \\
Admission diastolic BP, mm Hg & $89 \pm 14$ & $89 \pm 21$ & $0.998^{\mathrm{a}}$ \\
Diabetes mellitus & $31.4 \%$ & $43 \%$ & $0.301^{\mathrm{b}}$ \\
$\quad$ Admission glucose level, mg/dL & $136.9 \pm 46.5$ & $137 \pm 74$ & $0.992^{\mathrm{a}}$ \\
Hyperlipidemia, mg/dL & $57 \%$ & $67.6 \%$ & $0.361^{\mathrm{b}}$ \\
$\quad$ LDL, mg/dL & $100 \pm 37$ & $107 \pm 44$ & $0.493^{\mathrm{a}}$ \\
$\quad$ HDL, mg/dL & $40.3 \pm 13$ & $48.8 \pm 21$ & $0.052^{\mathrm{a}}$ \\
$\quad$ Triglyceride, mg/dL & $100.9 \pm 45$ & $155.2 \pm 90$ & $0.003^{\mathrm{a}}$ \\
$\quad$ Cholesterol, mg/dL & $161 \pm 41$ & $186 \pm 51$ & $0.03^{\mathrm{a}}$ \\
Coronary artery disease & $20 \%$ & $10.8 \%$ & $0.279^{\mathrm{b}}$ \\
Congestive heart failure & $20 \%$ & $19 \%$ & $0.908^{\mathrm{b}}$ \\
Atrial fibrillation & $42.8 \%$ & $18.9 \%$ & $0.028^{\mathrm{b}}$ \\
Intravenous tPA rate & $51.4 \%$ & $27 \%$ & $0.034^{\mathrm{b}}$ \\
Time from stroke onset to MRI, min & $295 \pm 138$ & $339 \pm 161$ & $0.224^{\mathrm{a}}$ \\
MRI turnaround time, min & $90 \pm 33$ & $94 \pm 55$ & $0.701^{\mathrm{a}}$ \\
Mortality & $14.3 \%$ & $5.4 \%$ & $0.254^{\mathrm{d}}$ \\
Vessel occlusion & $91.4 \%$ & $18.9 \%$ & $0.000^{\mathrm{b}}$ \\
Perfusion-diffusion mismatch & $85.7 \%$ & $56.8 \%$ & $0.007^{\mathrm{b}}$ \\
Clinical diffusion mismatch & $14.3 \%$ & $43.2 \%$ & $0.000^{\mathrm{b}}$ \\
Endovascular therapy & $71.4 \%$ & $0 \%$ & \\
\hline & & & \\
\hline
\end{tabular}

BP, blood pressure; HDL, high-density lipoprotein; LDL, low-density lipoprotein; NIHSS, National In-

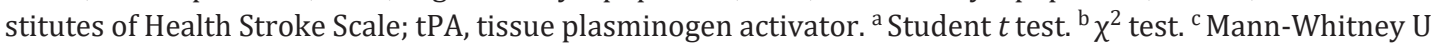
test. ${ }^{\mathrm{d}}$ Fisher exact test.

\section{Discussion}

In this observational retrospective study, we found that MRI selection for ET in AIS patients, including perfusion imaging, is feasible in a single large academic medical center. However, the MRI turnaround time was found to be $95.5 \pm 48.5 \mathrm{~min}$ and the total MRI time $27.7 \pm 12.8 \mathrm{~min}$, which appears to be higher than for CT screening only in other reported studies. In a study utilizing MRI screening prior to intravenous tPA in AIS, the door to needle time was found to be $85 \mathrm{~min}$ (73-102 $\mathrm{min}$ ) in the MRI screening group, $21 \mathrm{~min}$ longer than for CT screening only [6]. The trial utilized multimodal MRI requiring approximately $20 \mathrm{~min}$ in the scanner, including DWI, PWI, gradient echo, and magnetic resonance angiography.

From the experience at our tertiary academic medical center, the steps in time required from order to MRI are a significant source of delay and include MRI availability at the time of acute stroke and MRI screening from completion, especially when the patient is unable to give a history and a family member or proxy is not available immediately. The MRI technologist was available in-house at all time at our facility and should not have been a cause for delay.

In most centers, screening a candidate for ET utilizes CT/CT angiography. If a perfusion study is warranted, performing a perfusion CT should be less complicated than transporting the patient to MRI. In our data, we found that the turnaround time for multimodality MRI 
Table 3. Comparison of baseline clinical and demographic variables between subgroups with and without mismatch in AIS patients with vessel occlusion

\begin{tabular}{|c|c|c|c|}
\hline & Mismatch & No mismatch & Significance \\
\hline Number of patients & 32 & 7 & \\
\hline Age, years & $64.4 \pm 15.6$ & $76.7 \pm 7.2$ & $0.0495^{\mathrm{a}}$ \\
\hline Male sex & $56 \%$ & $28.5 \%$ & $0.235^{c}$ \\
\hline Median admission NIHSS score & $17.5(12-21)$ & 19 (16-22) & $0.474^{\mathrm{b}}$ \\
\hline Hypertension & $78 \%$ & $100 \%$ & $0.313^{\mathrm{c}}$ \\
\hline Admission systolic BP, mm Hg & $158 \pm 30$ & $150 \pm 19$ & $0.529^{\mathrm{a}}$ \\
\hline Admission diastolic $\mathrm{BP}, \mathrm{mm} \mathrm{Hg}$ & $89.5 \pm 14$ & $91 \pm 19$ & $0.813^{\mathrm{a}}$ \\
\hline Diabetes mellitus & $31 \%$ & $57 \%$ & $0.225^{c}$ \\
\hline Admission glucose level, mg/dL & $138 \pm 47$ & $114 \pm 21$ & $0.201^{\mathrm{a}}$ \\
\hline Hyperlipidemia & $57 \%$ & $71 \%$ & $0.438^{\mathrm{c}}$ \\
\hline $\mathrm{LDL}, \mathrm{mg} / \mathrm{dL}$ & $95.7 \pm 34.4$ & $125 \pm 40$ & $0.071^{\mathrm{a}}$ \\
\hline $\mathrm{HDL}, \mathrm{mg} / \mathrm{dL}$ & $40.3 \pm 12$ & $46 \pm 16$ & $0.33^{\mathrm{a}}$ \\
\hline Triglyceride, mg/dL & $100.3 \pm 45$ & $126.8 \pm 35.5$ & $0.182^{\mathrm{a}}$ \\
\hline Cholesterol, mg/dL & $156 \pm 40$ & $196 \pm 48$ & $0.035^{\mathrm{a}}$ \\
\hline Coronary artery disease & $19 \%$ & $28.6 \%$ & $0.617^{c}$ \\
\hline Congestive heart failure & $22 \%$ & $71.4 \%$ & $0.02^{\mathrm{c}}$ \\
\hline Atrial fibrillation & $43.8 \%$ & $14.3 \%$ & $0.216^{\mathrm{c}}$ \\
\hline Time from stroke onset to MRI, min & $290 \pm 141$ & $300 \pm 147$ & $0.877^{\mathrm{a}}$ \\
\hline MRI turnaround time, min & $92 \pm 33$ & $87 \pm 38$ & $0.758^{\mathrm{a}}$ \\
\hline Mortality & $15.6 \%$ & $28.6 \%$ & $0.588^{\mathrm{c}}$ \\
\hline Perfusion-diffusion mismatch & $84 \%$ & $57 \%$ & $0.137^{c}$ \\
\hline Clinical diffusion mismatch & $16 \%$ & $43 \%$ & \\
\hline \multicolumn{4}{|l|}{ DWI lesion volume } \\
\hline None to small & $20(62.5 \%)$ & $1(14.3 \%)$ & \\
\hline Medium to large & $12(37.5 \%)$ & $6(85.7 \%)$ & $0.038^{\mathrm{c}}$ \\
\hline \multicolumn{4}{|l|}{ Vessel occlusion site } \\
\hline Anterior & $31(96.8 \%)$ & $5(71 \%)$ & $0.077^{\mathrm{c}}$ \\
\hline Posterior & $1(3.2 \%)$ & $2(2.9 \%)$ & \\
\hline
\end{tabular}

AIS, acute ischemic stroke; BP, blood pressure; HDL, high-density lipoprotein; LDL, low-density lipo-

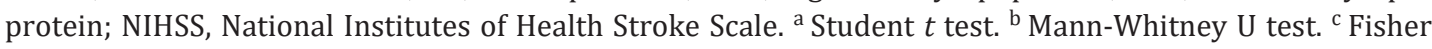
exact test.

screening in our high-volume academic medical center setting was $95.5 \mathrm{~min}$. The current goal for door to puncture time per American Heart Association (AHA) guidelines is $<90$ min for acute anterior circulation large vessel occlusion ischemic stroke and the imaging recommendation only includes CT/CT angiography or magnetic resonance angiography. The benefit of imaging with perfusion CT or DWI and PWI per AHA guidelines is unknown [8]. MRI selection may not provide more benefit in patients with acute vessel occlusion within a short time from stroke $(<6 \mathrm{~h})$ as per current AHA guidelines [8]. The chances of these patients to have treatable mismatch are very high and they will benefit from recanalization as early as possible. However, in the setting of ET in patients beyond these hours in whom benefit is likely dependent on salvageable penumbra, imaging that can sufficiently evaluate penumbra is needed.

Penumbra can be studied using a number of different methodologies including CT, MRI, PET, SPECT, and molecular imaging. A proposed MRI signature of penumbra is a mismatch between the area of abnormality on PWI and DWI. In patients with decrease salvageable penumbra, ET may not lead to a better functional outcome per the DEFUSE 2 trial [9]. Currently, the most important use for penumbra evaluation is for patient selection for ET beyond the 6-h window as per the recently published DAWN trial [4]. However, in the setting 
of hyperacute stroke where "time is brain," a fast and effective imaging modality is preferred. Multimodality MRI (including PWI) was found to have an average turnaround time of $96 \mathrm{~min}$ in our study, including $27 \mathrm{~min}$ in the MRI scanner, which is comparable to the reported time in the MRI scanner of approximately $20 \mathrm{~min}$ in another study [6]. Utilizing CDM for ET selection with MRI DWI sequence only and foregoing PWI might be a better time-saving option for most center as per DAWN trial protocol.

In our study, 32 of 39 patients with vessel occlusion were found to have mismatch. There were 7 patients who did not have mismatch in the presence of vessel occlusion likely due to poor collateral circulation and early expansion of the infarct. These patients were older and had a high total serum cholesterol level as well as frequent history of CHF. Thus, it is likely that hypercholesterolemia and CHF resulted in early expansion of infarction, making mismatch less likely to be detected as the time from stroke onset increased. This further emphasizes the concept of "time is brain," as salvageable brain tissue disappears faster in some patients with older age, hypercholesterolemia, and CHF.

MRI in AIS will exclude patients who have stroke mimics and do not need ET. Stroke mimics were reported to be $3-11 \%$ in patients presenting with AIS symptom [10]. In our study group, the proportion of stroke mimics was 19\%, which was slightly higher than in other reports. The differential of stroke mimics may not be straightforward since they often share similar risk factors as AIS, except for younger age, as shown in our study. Utilizing MRI for selection also significantly improved the accuracy of diagnosing stroke from noncontrast CT/CT angiography [11]. None of the patients in our study received unnecessary intravenous tPA.

MRI in AIS will exclude patients who are likely to be harmed. Patients with an infarct core lesion (DWI volume) $>100 \mathrm{~mL}$ or $\mathrm{PWI}>100 \mathrm{~mL}$ with a $\mathrm{T}_{\max }>8 \mathrm{~s}$ were considered to have malignant mismatch $[7,12]$. This is approximately greater than one-third of the volume of the MCA territory and is found to have high risk of ICH. In our study, among the 10 patients with mismatch who were not triaged to ET, 3 were found to have DWI lesions greater than onethird of the volume of the MCA territory. This was about $8.6 \%$ of all patients who had mismatch. Recent studies applied a DWI lesion volume of $70 \mathrm{~cm}^{3}$ as the cutoff for ET, as it was shown that a DWI lesion volume $>70 \mathrm{~cm}^{3}$ had a high specificity for poor outcome with or without therapy [13]. Yoo et al. [13] showed that about $17.6 \%$ of patients with mismatch had a DWI volume $>70 \mathrm{~cm}^{3}$. Thus, it is expected that about $10-18 \%$ of patients with mismatch will be excluded from ET due to large DWI lesion on MRI. Furthermore, Leslie-Mazwi et al. [14] described MRI criteria using DWI volume and clinical criteria to classify patients with ICA or MCA occlusion likely to benefit from ET, which could be implemented for triage.

The limitation of this study is the fact that is was a retrospectively case series study without control patients for ET. Selection bias or recall bias might have affected the results. The relatively small number of patients who received ET did not allow further statistical analysis. The ET method was not conventional with stent-retriever routinely used to achieve reperfusion in clinic practice, as the study was carried out from 2008 to 2010.

In conclusion, multimodality MRI screening for AIS symptoms for ET is feasible. Optimizing each center's protocol and the utilization of MRI with DWI only may be a time-saving alternative.

\section{Disclosure Statement}

The authors have nothing to disclose. 


\section{References}

1 Goyal M, Menon BK, van Zwam WH, Dippel DW, Mitchell PJ, Demchuk AM, Dávalos A, Majoie CB, van der Lugt A, de Miquel MA, Donnan GA, Roos YB, Bonafe A, Jahan R, Diener HC, van den Berg LA, Levy EI, Berkhemer OA, Pereira VM, Rempel J, Millán M, Davis SM, Roy D, Thornton J, Román LS, Ribó M, Beumer D, Stouch B, Brown S, Campbell BC, van Oostenbrugge RJ, Saver JL, Hill MD, Jovin TG; HERMES collaborators: Endovascular thrombectomy after large-vessel ischaemic stroke: a meta-analysis of individual patient data from five randomised trials. Lancet 2016;387:1723-1731.

2 Astrup J, Symon L, Branston NM, Lassen NA: Cortical evoked potential and extracellular $\mathrm{K}^{+}$and $\mathrm{H}^{+}$at critical levels of brain ischemia. Stroke 1977;8:51-57.

3 Jovin TG, Liebeskind DS, Gupta R, Rymer M, Rai A, Zaidat 00, Abou-Chebl A, Baxter B, Levy EI, Barreto A, Nogueira RG: Imaging-based endovascular therapy for acute ischemic stroke due to proximal intracranial anterior circulation occlusion treated beyond $8 \mathrm{~h}$ from time last seen well: retrospective multicenter analysis of 237 consecutive patients. Stroke 2011;42:2206-2211.

4 Nogueira RG, Jadhav AP, Haussen DC, Bonafe A, Budzik RF, Bhuva P, Yavagal DR, Ribo M, Cognard C, Hanel RA, Sila CA, Hassan AE, Millan M, Levy EI, Mitchell P, Chen M, English JD, Shah QA, Silver FL, Pereira VM, Mehta BP, Baxter BW, Abraham MG, Cardona P, Veznedaroglu E, Hellinger FR, Feng L, Kirmani JF, Lopes DK, Jankowitz BT, Frankel MR, Costalat V, Vora NA, Yoo AJ, Malik AM, Furlan AJ, Rubiera M, Aghaebrahim A, Olivot JM, Tekle WG, Shields R, Graves T, Lewis RJ, Smith WS, Liebeskind DS, Saver JL, Jovin TG; DAWN Trial Investigators: Thrombectomy 6 to 24 hours after stroke with a mismatch between deficit and infarct. N Engl J Med 2018; 378:11-21.

5 Vymazal J, Rulseh AM, Keller J, Janouskova L: Comparison of CT and MR imaging in ischemic stroke. Insights Imaging 2012;3:619-627.

6 Kang DW, Chalela JA, Dunn W, Warach S; NIH-Suburban Stroke Center Investigators: MRI screening before standard tissue plasminogen activator therapy is feasible and safe. Stroke 2005;36:1939-1943.

7 Albers GW, Thijs VN, Wechsler L, Kemp S, Schlaug G, Skalabrin E, Bammer R, Kakuda W, Lansberg MG, Shuaib A, Coplin W, Hamilton S, Moseley M, Marks MP; DEFUSE Investigators: Magnetic resonance imaging profiles predict clinical response to early reperfusion: the Diffusion and Perfusion Imaging Evaluation for Understanding Stroke Evolution (DEFUSE) study. Ann Neurol 2006;60:508-517.

8 Powers WJ, Derdeyn CP, Biller J, Coffey CS, Hoh BL, Jauch EC, Johnston KC, Johnston SC, Khalessi AA, Kidwell CS, Meschia JF, Ovbiagele B, Yavagal DR; American Heart Association Stroke Council: 2015 American Heart Association/American Stroke Association focused update of the 2013 guidelines for the early management of patients with acute ischemic stroke regarding endovascular treatment: a guideline for healthcare professionals from the American Heart Association/American Stroke Association. Stroke 2015;46:3020-3035.

9 Lansberg MG, Straka M, Kemp S, Mlynash M, Wechsler LR, Jovin TG, Wilder MJ, Lutsep HL, Czartoski TJ, Bernstein RA, Chang CW, Warach S, Fazekas F, Inoue M, Tipirneni A, Hamilton SA, Zaharchuk G, Marks MP, Bammer R, Albers GW; DEFUSE 2 study investigators: MRI profile and response to endovascular reperfusion after stroke (DEFUSE 2): a prospective cohort study. Lancet Neurol 2012;11:860-867.

10 Chernyshev OY, Martin-Schild S, Albright KC, Barreto A, Misra V, Acosta I, Grotta JC, Savitz SI: Safety of tPA in stroke mimics and neuroimaging-negative cerebral ischemia. Neurology 2010;74:1340-1345.

11 Gonzalez RG, Schaefer PW, Buonanno FS, Schwamm LH, Budzik RF, Rordorf G, Wang B, Sorensen AG, Koroshetz WJ: Diffusion-weighted MR imaging: diagnostic accuracy in patients imaged within 6 hours of stroke symptom onset. Radiology 1999;210:155-162.

12 Davis SM, Donnan GA, Parsons MW, Levi C, Butcher KS, Peeters A, Barber PA, Bladin C, De Silva DA, Byrnes G, Chalk JB, Fink JN, Kimber TE, Schultz D, Hand PJ, Frayne J, Hankey G, Muir K, Gerraty R, Tress BM, Desmond PM; EPITHET investigators: Effects of alteplase beyond $3 \mathrm{~h}$ after stroke in the Echoplanar Imaging Thrombolytic Evaluation Trial (EPITHET): a placebo-controlled randomised trial. Lancet Neurol 2008;7:299-309.

13 Yoo AJ, Verduzco LA, Schaefer PW, Hirsch JA, Rabinov JD, Gonzalez RG: MRI-based selection for intra-arterial stroke therapy: value of pretreatment diffusion-weighted imaging lesion volume in selecting patients with acute stroke who will benefit from early recanalization. Stroke 2009;40:2046-2054.

14 Leslie-Mazwi TM, Hirsch JA, Falcone GJ, Schaefer PW, Lev MH, Rabinov JD, Rost NS, Schwamm L, González RG: Endovascular stroke treatment outcomes after patient selection based on magnetic resonance imaging and clinical criteria. JAMA Neurol 2016;73:43-49. 